\title{
ON BIVARIATE GAUSSIAN CUBATURE FORMULAE
}

\author{
H. J. SCHMID AND YUAN XU \\ (Communicated by J. Marshall Ash) \\ Dedicated to Professor I. P. Mysovskikh
}

\begin{abstract}
It is shown that for two classes of integrals the results of Gaussian quadrature can be extended straightforwardly to the bivariate case. For these classes Gaussian formulae of an arbitrary degree are derived.
\end{abstract}

\section{INTRODUCTION}

Let $\Pi_{n}^{d}$ be the set of polynomials of total degree $n$ in $d$ variables and $\Pi^{d}$ the set of all polynomials in $d$ variables. For a nonnegative function $W$ on $\mathbb{R}^{d}$ a minimal cubature formula of degree $m$ is a linear functional

$$
\mathscr{I}_{m}(f)=\sum_{k=1}^{N} \lambda_{k} f\left(\mathbf{x}_{k}\right), \quad \lambda_{k}>0, \mathbf{x}_{k} \in \mathbb{R}^{d},
$$

where $N$-the number of the involved nodes $x_{k}$-is minimal, such that

$$
\int_{\mathbf{R}} f(\mathbf{x}) W(\mathbf{x}) d \mathbf{x}=\mathscr{I}_{m}(f), \quad \forall f \in \Pi_{m}^{d} .
$$

It is known that $N \geq \operatorname{dim} \Pi_{[m / 2]}^{d}$ in general. Formulae for which the equality holds are of the highest precision, just like the classical Gaussian quadrature formulae, and we shall term them Gaussian cubatures. For $d=1$ the results of Gaussian quadrature are well known (cf. [4]). If $\left\{p_{k}\right\}$ are the orthonormal polynomials with respect to the weight function, then the $N=\operatorname{dim} \Pi_{n-1}^{1}=n$ roots of

$$
p_{n}+\rho p_{n-1}, \quad \rho \in \mathbb{R},
$$

are the nodes of a minimal quadrature rule of degree $2 n-2$. Moreover, for $\rho=0$ a uniquely determined formula of degree $2 n-1$ will be obtained. A straightforward extension of these results for higher dimensions is not possible in general. Möller [11, 12] proved for centrally symmetric weight functions, i.e., $\int x^{k-j} y^{j} W(x, y) d x d y=0$ for odd $k, 0 \leq j \leq k$, that $N=\operatorname{dim} \Pi_{n-1}^{2}$

Received by the editors April 16, 1992 and, in revised form, January 4, 1993.

1991 Mathematics Subject Classification. Primary 65D30, 41 A05.

Key words and phrases. Gaussian cubature, bivariate orthogonal polynomial, common zeros of orthogonal polynomials. 
nodes do not suffice to obtain degree $2 n-1$. The same behaviour was found for Jacobi weight furctions on the simplex [1, 2].

For $d=2$ the existence of minimal formulae of degree $2 n-1$ with $\operatorname{dim} \Pi_{n-1}^{2}$ nodes has been characterized by Mysovskikh [15]. The nodes of such a formula, if it exists, are the common zeros of bivariate orthogonal polynomials. Mysovskikh and Černicina [13] constructed a special weight function and an associated formula for Radon's case, i.e., degree 5. The existence of linear functionals admitting formulae of the discussed type was studied by Kuz'menkov [10]. Since then one was sure that such functionals would be exotic and Gaussian cubatures would be rare.

The main purpose of this paper is to present two classes of integrals for which Mysovskikh's characterization holds and to extend the one-dimensional results directly to the bivariate case. The integrals discussed allow an explicit computation of minimal formulae of an arbitrary even or odd degree of exactness. Since only a few results on this topic are known, our examples might be of general interest.

Let $w(x)$ be a nonnegative function on $\mathbb{R}$. Let $\left\{p_{n}\right\}$ be orthonormal polynomials with respect to $w$, and let $x_{k, n}$ be the zeros of $p_{n}+\rho p_{n-1}$, where $\rho$ is an arbitrary but fixed real number. The roots are ordered by $x_{1, n}<\cdots<x_{n, n}$.

Theorem 1. Let $w$ be a nonnegative function on an interval $I$. Let $u=x+y$ and $v=x y$, and define $W(u, v)=w(x) w(y)$. Then we have the following Gaussian cubatures of degree $2 n-2$ :

$$
\begin{array}{r}
\int_{R} \int_{R} f(u, v) W(u, v)\left(u^{2}-4 v\right)^{-1 / 2} d u d v \\
=\sum_{k=1}^{n} \sum_{j=1}^{k} \lambda_{j, k} f\left(x_{k, n}+x_{j, n}, x_{k, n} x_{j, n}\right)
\end{array}
$$

and

$$
\begin{aligned}
& \iint_{R} f(u, v) W(u, v)\left(u^{2}-4 v\right)^{1 / 2} d u d v \\
& =\sum_{k=1}^{n+1} \sum_{j=1}^{k-1} \lambda_{k, j} f\left(x_{k, n+1}+x_{j, n+1}, x_{k, n+1} x_{j, n+1}\right),
\end{aligned}
$$

where the integral is over the region $R=\{(u, v) \mid(x, y) \in I \times I$ and $x<y\}$. If $\rho=0$, then a uniquely determined formula of degree $2 n-1$ will be obtained.

Our proof, in the following section, is based on the bivariate orthogonal polynomials introduced by Koornwinder in [7]. For a given $n \in \mathbb{N}_{0}$ let $u=x+y$ and $v=x y$ and define

$$
\begin{gathered}
P_{k}^{n,(-1 / 2)}(u, v)= \begin{cases}p_{n}(x) p_{k}(y)+p_{n}(y) p_{k}(x) & \text { if } k<n, \\
\sqrt{2} p_{n}(x) p_{n}(y) & \text { if } k=n\end{cases} \\
P_{k}^{n,(1 / 2)}(u, v)=\frac{p_{n+1}(x) p_{k}(y)-p_{n+1}(y) p_{k}(x)}{x-y} .
\end{gathered}
$$

Then $P_{k}^{n,( \pm 1 / 2)}$ are polynomials of total degree $n$. Koornwinder [7, p. 468] showed that $\left\{P_{k}^{n,( \pm 1 / 2)}\right\}$ are bivariate orthogonal systems with respect to the 


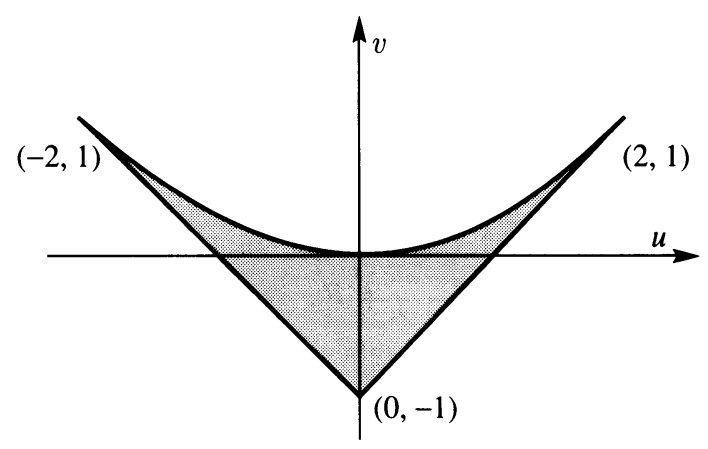

Figure 1

weight function $\left(u^{2}-4 v\right)^{ \pm 1 / 2} W(u, v)$. For $I=[-1,1]$ the region $R$ on the $(u, v)$ plane is given in Figure 1 .

In [8] Koornwinder defined and investigated an important class of bivariate orthogonal polynomials $\left\{P_{k}^{n,(\gamma)}\left(w^{(\alpha, \beta)}\right)\right\}$ that are orthogonal with respect to the weight function $\left(u^{2}-4 v\right)^{\gamma} W(u, v)$, where $W(u, v)=w^{(\alpha, \beta)}(x) w^{(\alpha, \beta)}(y)$ and $w^{(\alpha, \beta)}=(1-x)^{\alpha}(1+x)^{\beta}$ is the Jacobi weight (see [9, 20] for further analysis; the polynomials are denoted $P_{k, n}^{\alpha, \beta, \gamma}$ in [8]). The cases $\gamma= \pm \frac{1}{2}$ correspond to our $P_{k}^{n,( \pm 1 / 2)}$ with $w$ being a Jacobi weight. It is natural to ask whether the weight function $\left(u^{2}-4 v\right)^{\gamma} W(u, v)$ leads to Gaussian cubatures for $\gamma \neq \pm \frac{1}{2}$. In [20, (10.7)-(10.10), p. 518] (see also [9, (3.21), (3.22), p. 465]) $P_{k}^{n,(\gamma)}\left(w^{( \pm 1.2, \pm 1 / 2)}\right)$ are given explicitly in terms of Gegenbauer polynomials. Using these formulae, we can prove that in these four cases the answer is negative, at least for the odd degree cubature. That is, for $w^{( \pm 1 / 2, \pm 1 / 2)}$ the weight function $\left(u^{2}-4 v\right)^{\gamma} W(u, v)$ leads to Gaussian cubature of degree $2 n-1$ only if $\gamma= \pm \frac{1}{2}$.

\section{ProOF}

For a given weight function $W$ on $\mathbb{R}^{2}$ let $\left\{P_{k}^{n}\right\}_{k=0}^{n} \underset{n=0}{\infty}$ be a sequence of orthonormal polynomials corresponding to $W$, where the superscript $n$ means that $P_{k}^{n}$ is of total degree $n$. Using the vector notation

$$
\mathbb{P}_{n}(\mathbf{x})=\left[P_{0}^{n}(\mathbf{x}), P_{1}^{n}(\mathbf{x}), \ldots, P_{n}^{n}(\mathbf{x})\right]^{\mathrm{T}}, \quad \mathbf{x}=(x, y),
$$

the orthonormal property of $\left\{P_{k}^{n}\right\}$ is described by

$$
\int_{\mathbf{R}^{2}} \mathbb{P}_{n}(\mathbf{x}) \mathbb{P}_{m}^{\mathrm{T}}(\mathbf{x}) W(\mathbf{x}) d x d y=\delta_{m, n} E_{n+1},
$$

where $E_{n}$ denotes the $n \times n$ identity matrix. Throughout this paper, the notation $A: i \times j$ means that $A$ is a matrix of size $i \times j$. For convenience, we sometimes call $\mathbb{P}_{n}$ orthonormal polynomials. Using this vector notation, many properties of the univariate orthogonal polynomials have been extended to the multivariate setting (cf. [5, 6, 21-25]). In particular, a system of orthonormal polynomials satisfies a three-term relation

$$
x_{i} \mathbb{P}_{n}=A_{n, i} \mathbb{P}_{n+1}+B_{n, i} \mathbb{P}_{n}+A_{n-1}^{\mathrm{T}}, \mathbb{P}_{n-1}, \quad i=1,2,
$$


where $A_{n, i}:(n+1) \times(n+2)$ and $B_{n, i}:(n+1) \times(n+1)$ are matrices satisfying

$$
\begin{aligned}
A_{n, 1} A_{n+1,2} & =A_{n, 2} A_{n+1,1}, \\
A_{n, 1} B_{n+1,2}+B_{n, 1} A_{n, 2} & =B_{n, 2} A_{n, 1}+A_{n, 2} B_{n+1,1},
\end{aligned}
$$

and

$$
\begin{aligned}
& A_{n-1,1}^{\mathrm{T}} A_{n-1,2}+B_{n, 1} B_{n, 2}+A_{n, 1} A_{n, 2}^{\mathrm{T}} \\
& \quad=A_{n-1,2}^{\mathrm{T}} A_{n-1,1}+B_{n, 2} B_{n, 1}+A_{n, 2} A_{n, 1}^{\mathrm{T}} .
\end{aligned}
$$

In addition, if a sequence of polynomials satisfies (2.2)-(2.5) for some matrices $A_{n, i}$ and $B_{n, i}$ and $\operatorname{rank}\left(A_{n, 1}^{\mathrm{T}} \mid A_{n, 2}^{\mathrm{T}}\right)^{\mathrm{T}}=n+2$, then it is orthonormal with respect to a square positive linear functional (Favard's theorem [21, 22]). The common zeros of the components in $\mathbb{P}_{n}$ will be called zeros of $\mathbb{P}_{n}$.

Theorem 2. For a given weight function $W$ a Gaussian cubature of degree $2 n-2$ exists if and only if there is a matrix $\Gamma: n+1 \times n$ such that $\mathbb{P}_{n}+\Gamma \mathbb{P}_{n-1}$ has $\operatorname{dim} \Pi_{n-1}^{2}$ zeros. These zeros are the notes of the cubature. Moreover, $\mathbb{P}_{n}+\Gamma \mathbb{P}_{n-1}$ has $\operatorname{dim} \Pi_{n-1}^{2}$ zeros if and only if

$$
A_{n-1,2} \Gamma A_{n-2,1}^{\mathrm{T}}=A_{n-1,1} \Gamma A_{n-2,2}^{\mathrm{T}}
$$

and

$$
\begin{aligned}
& {\left[A_{n-1,2} B_{n, 1}-A_{n-1,1} B_{n, 2}+A_{n-1,2} \Gamma A_{n-1,1}-A_{n-1,1} \Gamma A_{n-1,2}\right] \Gamma} \\
& \quad=A_{n-1,2} A_{n-1,1}^{\mathrm{T}}-A_{n-1,1} A_{n-1,2}^{\mathrm{T}}+A_{n-1,2} \Gamma B_{n-1,1}-A_{n-1,1} \Gamma B_{n-1,2} .
\end{aligned}
$$

If $\Gamma=0$ satisfies (2.6) and (2.7), then the degree of exactness is $2 n-1$.

This is the characterization of [17-19, 26] in terms of orthonormal polynomials. If $\Gamma=0$, then the theorem is reduced to Mysovskikh's characterization [15], i.e.,

$$
A_{n-1,2} A_{n-1,1}^{\mathrm{T}}=A_{n-1,1} A_{n-1,2}^{\mathrm{T}} \text {. }
$$

The formulation of this in [15] is in terms of a monic orthogonal basis; a simple proof in the present form can be found in [24].

In the following we shall derive the three-term relation satisfied by $\left\{P_{k}^{n,( \pm 1 / 2)}\right\}$, in vector notation, $\mathbb{P}_{n}^{( \pm 1 / 2)}$, and study conditions (2.6) and (27).

We denote the coefficient matrices in the three-term relation of $\mathbb{P}_{n}^{( \pm 1 / 2)}$ by $A_{n, i}^{( \pm 1 / 2)}$ and $B_{n, i}^{( \pm 1 / 2)}$. Let the weight function $w$ satisfy $\int w(x) d x=1$. Then the corresponding orthonormal polynomials $\left\{p_{n}\right\}$ satisfy a three-term relation given by (cf. [3])

$$
x p_{n}=a_{n} p_{n+1}+b_{n} p_{n}+a_{n-1} p_{n-1}, \quad n \geq 0,
$$

where $p_{0}=1$ and $p_{-1}=0$.

We consider $P_{k}^{n,(-1 / 2)}$ first and shall omit the superscript $-\frac{1}{2}$ in the following computation. From (1.5) and $u=x+y$ we have for $k<n$

$$
\begin{aligned}
u P_{k}^{n}(u, v)= & (x+y)\left(p_{n}(x) p_{k}(y)+p_{n}(y) p_{k}(x)\right) \\
= & a_{n}\left(p_{n+1}(x) p_{k}(y)+p_{n+1}(y) p_{k}(x)\right)+b_{n}\left(p_{n}(x) p_{k}(y)+p_{n}(y) p_{k}(x)\right) \\
& +a_{n-1}\left(p_{n-1}(x) p_{k}(y)+p_{n-1}(y) p_{k}(x)\right)+a_{k}\left(p_{k+1}(x) p_{n}(y)\right. \\
& \left.+p_{k+1}(y) p_{n}(x)\right)+b_{k}\left(p_{k}(x) p_{n}(y)+p_{k}(y) p_{n}(x)\right) \\
& +a_{k-1}\left(p_{k-1}(x) p_{n}(y)+p_{k-1}(y) p_{n}(x)\right) .
\end{aligned}
$$


Therefore, we have for $0 \leq k \leq n-2$

$$
u P_{k}^{n}=a_{n} P_{k}^{n+1}+b_{n} P_{k}^{n}+a_{n-1} P_{k}^{n-1}+a_{k} P_{k+1}^{n}+b_{k} P_{k}^{n}+a_{k-1} P_{k-1}^{n},
$$

for $k=n-1$

$$
u P_{n-1}^{n}=a_{n} P_{n-1}^{n+1}+b_{n} P_{n-1}^{n}+\sqrt{2} a_{n-1} P_{n-1}^{n-1}+\sqrt{2} a_{n-1} P_{n}^{n}+b_{n-1} P_{n-1}^{n}+a_{n-2} P_{n-2}^{n},
$$

and for $k=n$

$$
u P_{n}^{n}=\sqrt{2}(x+y) p_{n}(x) p_{n}(y)=\sqrt{2} a_{n} P_{n}^{n+1}+2 b_{n} P_{n}^{n}+\sqrt{2} a_{n-1} P_{n-1}^{n} .
$$

It follows from these formulas that

$$
\begin{aligned}
& A_{n, 1}^{(-1 / 2)}=a_{n}\left[\begin{array}{ccccc}
1 & & & \bigcirc & 0 \\
& \ddots & & & \vdots \\
& & 1 & & 0 \\
& & & \sqrt{2} & 0
\end{array}\right] \text {, } \\
& B_{n, 1}^{(-1 / 2)}=\left[\begin{array}{ccccc}
b_{0} & a_{0} & & & \bigcirc \\
a_{0} & b_{1} & a_{1} & & \\
& \ddots & \ddots & \ddots & \\
& & a_{n-2} & b_{n-1} & \sqrt{2} a_{n-1} \\
\bigcirc & & & \sqrt{2} a_{n-1} & b_{n}
\end{array}\right]+b_{n} E_{n+1} \text {. }
\end{aligned}
$$

Similarly, we have from (1.5), (2.9), and $v=x y$ that

$$
\begin{aligned}
v P_{k}^{n}(u, v)= & (x y)\left(p_{n}(x) p_{k}(y)+p_{n}(y) p_{k}(x)\right) \\
= & \left(a_{n} p_{n+1}(x)+b_{n} p_{n}(x)+a_{n-1} p_{n-1}(x)\right) \\
& \times\left(a_{k} p_{k+1}(y)+b_{k} p_{k}(y)+a_{k-1} p_{k-1}(y)\right) \\
& +\left(a_{n} p_{n+1}(y)+b_{n} p_{n}(y)+a_{n-1} p_{n-1}(y)\right) \\
& \times\left(a_{k} p_{k+1}(x)+b_{k} p_{k}(x)+a_{k-1} p_{k-1}(x)\right) .
\end{aligned}
$$

Therefore, for $0 \leq k \leq n-2$ we have

$$
\begin{aligned}
v P_{k}^{n}= & a_{n}\left(a_{k} P_{k+1}^{n+1}+b_{k} P_{k}^{n+1}+a_{k-1} P_{k-1}^{n+1}\right) \\
& +b_{n}\left(a_{k} P_{k+1}^{n}+b_{k} P_{k}^{n}+a_{k-1} P_{k-1}^{n}\right) \\
& +a_{n-1}\left(a_{k} P_{k+1}^{n-1}+b_{k} P_{k}^{n-1}+a_{k-1} P_{k-1}^{n-1}\right),
\end{aligned}
$$

where for $k=n-2$ the coefficient of $P_{n-1}^{n-1}$ is multiplied by $\sqrt{2}$, for $k=n-1$

$$
\begin{aligned}
v P_{n-1}^{n}= & a_{n}\left(a_{n-1} P_{n}^{n+1}+b_{n-1} P_{n-1}^{n+1}+a_{n-2} P_{n-2}^{n+1}\right) \\
& +b_{n}\left(\sqrt{2} a_{n-1} P_{n}^{n}+b_{n-1} P_{n-1}^{n}+a_{n-2} P_{n-2}^{n}\right) \\
& +a_{n-1}^{2} P_{n-1}^{n}+a_{n-1}\left(\sqrt{2} b_{n-1} P_{n-1}^{n-1}+a_{n-2} P_{n-2}^{n-1}\right)
\end{aligned}
$$

and for $k=n$

$$
\begin{aligned}
v P_{n}^{n}= & \sqrt{2} x y p_{n}(x) p_{n}(y) \\
= & a_{n}\left(a_{n} P_{n+1}^{n+1}+\sqrt{2} b_{n} P_{n}^{n+1}+\sqrt{2} a_{n-1} P_{n-1}^{n+1}\right) \\
& +b_{n}\left(b_{n} P_{n}^{n}+\sqrt{2} a_{n-1} P_{n-1}^{n}\right)+a_{n-1} a_{n-1} P_{n-1}^{n-1} .
\end{aligned}
$$


It then follows that

$$
\begin{aligned}
A_{n, 2}^{(-1 / 2)}=a_{n} & {\left[\begin{array}{cccccc}
b_{0} & a_{0} & & & \bigcirc & 0 \\
a_{0} & b_{1} & a_{1} & & & \vdots \\
& \ddots & \ddots & \ddots & & \vdots \\
& & a_{n-2} & b_{n-1} & a_{n-1} & 0 \\
\bigcirc & & & \sqrt{2} a_{n-1} & \sqrt{2} b_{n} & a_{n}
\end{array}\right], } \\
B_{n, 2}^{(-1 / 2)}=b_{n} & {\left[\begin{array}{ccccc}
b_{0} & a_{0} & & & \bigcirc \\
a_{0} & b_{1} & a_{1} & & \\
& \ddots & \ddots & \ddots & \\
& & a_{n-2} & b_{n-1} & \sqrt{2} a_{n-1} \\
\bigcirc & & & \sqrt{2} a_{n-1} & b_{n}
\end{array}\right]+a_{n-1}^{2}\left[\begin{array}{ccc}
\bigcirc & & \\
1 & 0 \\
0 & 0
\end{array}\right] . }
\end{aligned}
$$

The three-term relation for $\mathbb{P}_{n}^{(1 / 2)}$ can be obtained similarly. The coefficient matrices are given as

$$
\begin{aligned}
& A_{n, 1}^{(1 / 2)}=a_{n+1}\left[\begin{array}{cccc}
1 & & \bigcirc & 0 \\
& \ddots & & \vdots \\
\bigcirc & & 1 & 0
\end{array}\right], \\
& B_{n, 1}^{(1 / 2)}=\left[\begin{array}{ccccc}
b_{0} & a_{0} & & & \bigcirc \\
a_{0} & b_{1} & a_{1} & & \\
& \ddots & \ddots & \ddots & \\
& & a_{n-2} & b_{n-1} & a_{n-1} \\
\bigcirc & & & a_{n-1} & b_{n}
\end{array}\right]+b_{n+1} E_{n+1} \text {. } \\
& A_{n, 2}^{(1 / 2)}=a_{n+1}\left[\begin{array}{cccccc}
b_{0} & a_{0} & & & \bigcirc & 0 \\
a_{0} & b_{1} & a_{1} & & & \vdots \\
& \ddots & \ddots & \ddots & & \vdots \\
& & a_{n-2} & b_{n-1} & a_{n-1} & 0 \\
\bigcirc & & & a_{n-1} & b_{n} & a_{n}
\end{array}\right] \text {, } \\
& B_{n, 2}^{(1 / 2)}=b_{n+1}\left[\begin{array}{ccccc}
b_{0} & a_{0} & & & \bigcirc \\
a_{0} & b_{1} & a_{1} & & \\
& \ddots & \ddots & \ddots & \\
& & a_{n-2} & b_{n-1} & a_{n-1} \\
\bigcirc & & & a_{n-1} & b_{n}
\end{array}\right]-a_{n}^{2}\left[\begin{array}{cc}
\bigcirc & \\
& 1
\end{array}\right] \text {. }
\end{aligned}
$$

From these matrices it follows easily that $A_{n, i}^{( \pm 1 / 2)}$ satisfy equation (2.8). Taking this into account and using equations (2.3)-(2.5), condition (2.7) can be rewritten as

$$
\begin{gathered}
{\left[B_{n-1,1} A_{n-1,2}-B_{n-1,2} A_{n-1,1}\right] \Gamma-A_{n-1,2} \Gamma B_{n-1,1}+A_{n-1,1} \Gamma B_{n-1,2}} \\
\quad=\left[A_{n-1,1} \Gamma A_{n-1,2}-A_{n-1,2} \Gamma A_{n-1,1}\right] \Gamma .
\end{gathered}
$$

Setting

$$
\Gamma^{(-1 / 2)}=\rho\left[\begin{array}{ccccc}
1 & & & 0 & 0 \\
& \ddots & & & \vdots \\
& & 1 & & 0 \\
0 & & \sqrt{2} & & -\rho
\end{array}\right]^{\mathrm{T}}
$$


and $\Gamma^{(1 / 2)}=\rho A_{n-1,1}^{(1 / 2) \mathrm{T}}, \rho \in \mathbb{R}$, it is not too hard to check that the $\Gamma^{( \pm 1 / 2)}$ satisfy the corresponding equation (2.6) and solve the corresponding matrix equation.

Thus, the existence of $\operatorname{dim} \Pi_{n-1}^{2}$ common zeros of $\mathbb{P}_{n}^{( \pm 1 / 2)}+\Gamma^{( \pm 1 / 2)} \mathbb{P}_{n-1}^{( \pm 1 / 2)}$ follows from Theorem 2. Moreover, from (1.5) we have

$$
\begin{aligned}
& P_{k}^{n,(-1 / 2)}(u, v)+\rho P_{k}^{n-1,(-1 / 2)}(u, v) \\
& \quad=\left[p_{n}(x)+\rho p_{n-1}(x)\right] p_{k}(y)+\left[p_{n}(y)+\rho p_{n-1}(y)\right] p_{k}(x), \quad k<n,
\end{aligned}
$$

and

$$
\begin{aligned}
& P_{n}^{n,(-1 / 2)}(u, v)-\rho^{2} P_{n}^{n-1,(-1 / 2)}(u, v) \\
& \quad=\sqrt{2}\left[p_{n}(x)+\rho p_{n-1}(x)\right] p_{n}(y)-\sqrt{2} \rho p_{n-1}(x)\left[p_{n}(y)+\rho p_{n-1}(y)\right] .
\end{aligned}
$$

Hence these polynomials vanish for $(x, y)=\left(x_{k, n}, x_{j, n}\right)$. Since the transformation $u=x+y$ and $v=x y$ is symmetric in $x$ and $y$, the distinct common zeros of $\mathbb{P}_{n}^{(-1 / 2)}+\Gamma_{n-1,1}^{(-1 / 2) \mathrm{T}} \mathbb{P}_{n-1}^{(1 / 2)}$ are given by $\left(x_{k, n}+x_{j, n}, x_{k, n} x_{j, n}\right)$ for $j \leq k$, which are the nodes in (1.3). Similarly, from (1.6) we obtain

$$
\begin{aligned}
& P_{k}^{n,(1 / 2)}(u, v)+\rho P_{k}^{n-1,(1 / 2)}(u, v) \\
& \quad=\frac{\left[p_{n+1}(x)+\rho p_{n}(x)\right] p_{k}(y)-\left[p_{n+1}(y)+\rho p_{n}(y)\right] p_{k}(x)}{x-y}, \quad k \leq n .
\end{aligned}
$$

The distinct common zeros of $\mathbb{P}_{n}^{(1 / 2)}+\Gamma^{(1 / 2)} \mathbb{P}_{n-1}^{(1 / 2)}$ are given by

$$
\left(x_{k, n+1}+x_{j, n+1}, x_{k, n+1} x_{j, n+1}\right), \quad j<k,
$$

which are nodes in (1.4).

We remark that in both cases all nodes are inside the domain of integration for $\rho$ in an open interval containing 0 . The end points of this interval can be determined explicitly using the one-dimensional theory. Furthermore, the degree of exactness is $2 n-2$, except if $\rho=0$, then we obtain degree $2 n-1$.

The coefficients $\lambda_{k, j}$ of the formulae obtained are positive since the number of nodes attains the lower bound $\operatorname{dim} \Pi_{n-1}^{2}$. Furthermore, it is known that the coefficients $\lambda_{k}$ in (1.1) are given by the values of the inverse of the reproducing kernel function at $\mathbf{x}_{k}$ (cf. [14]). So we get for (1.3)

$$
\lambda_{k, j}=1 / \sum_{k=0}^{n} \sum_{j=0}^{k}\left[P_{j}^{k,(-1 / 2)}\left(x_{k, n}+x_{j, n}, x_{k, n} x_{j, n}\right)\right]^{2} .
$$

For other expressions of $\lambda_{k}$ see [23].

Remark. Theorem 1 can be verified just by counting the number of real common zeros of $\mathbb{P}_{n}^{( \pm 1 / 2)}+\Gamma^{( \pm 1 / 2)} \mathbb{P}_{n-1}^{( \pm 1 / 2)}$ and taking into account the upper bound for the common real zeros of a fundamental system of polynomials (see [15]). However, this gives no further insight. We hope that the matrices in the threeterm relation and matrix equations (2.6) and (2.7) could be suggestive for more general cases, too. Theoretically, one can start with matrices $\left\{A_{n, i}\right\}$ and $\left\{B_{n, i}\right\}$ that satisfy (2.3)-(2.5), (2.8), and a rank condition and use them as coefficient matrices to generate orthogonal polynomials that lead to Gaussian cubatures; every Gaussian cubature could be derived in this way according to Favard's 
theorem [25]. The matrices given here are the only explicit examples known today.

\section{ACKNOWLEDGMENTS}

We thank Professor Hubert Berens for his invaluable comments and his constant encouragement.

\section{REFERENCES}

$1 \mathrm{H}$. Berens and H. J. Schmid, On the number of nodes of odd degree cubature formulae for integrals with Jacobi weights on a simplex, Numerical Integration (T. O. Espelid and A. Genz, eds.), Kluwer, Dordrecht, 1992, pp. 37-44.

2. H. Berens, H. J. Schmid, and Y. Xu, On twodimensional definite orthogonal systems and on a lower bound for the number of nodes of associated cubature formulae, SIAM J. Math. Anal. (to appear).

3. T. S. Chihara, An introduction to orthogonal polynomials, Math. Appl., vol. 13, Gordon and Breach, New York, 1978.

4. P. J. Davis and P. Rabinowitz, Methods of numerical integration, Academic Press, New York, 1975.

5. M. A. Kowalski, The recursion formulas for orthogonal polynomials in $n$ variables, SIAM J. Math. Anal. 13 (1982), 309-315.

6. __ Orthogonality and recursion formulas for polynomials in $n$ variables, SIAM J. Math. Anal. 13 (1982), 316-323.

7. T. Koornwinder, Two-variable analogues of the classical orthogonal polynomials, Theory and Applications of Special Functions (R. A. Askey, ed.), Academic Press, New York, 1975.

8. Orthogonal polynomials in two variables which are eigenfunctions of two algebraically independent partial differential operators. I, II, Proc. Kon. Nederl. Akad. Wetensch. 36 (1974), 48-66.

9. T. Koornwinder and I. Sprinkhuizen-Kuyper, Generalized power series expansions for a class of orthogonal polynomials in two variables, SIAM J. Math. Anal. 9 (1978), 457-483.

10. V. A. Kuz'menkov, The existence of cubature formulas with the least possible number of node, Zh. Vychisl. Mat. i Mat. Fiz. 16 (1976), 1337-1339.

11. H. M. Möller, Polynomideale und Kubaturformeln, Ph.D. Thesis, Univ. of Dortmund, 1973.

12. __ Kubaturformeln mit minimaler Knotenzahl, Numer. Math. 25 (1976), 185-200.

13. I. P. Mysovskikh and V. Ja. Černicina, Answer to a question of Radon, Dokl. Akad. Nauk SSSR 198 (1971), 537-539.

14. I. P. Mysovskikh, On the construction of cubature formulas with fewest nodes, Soviet Math. 9 (1968), 277-280.

15. _ Numerical characteristics of orthogonal polynomials in two variables, Vestnik Leningrad Univ. Math. 3 (1976), 323-332.

16. _ The approximation of multiple integrals by using interpolatory cubature formulae, Quantitative Approximation (R. A. DeVore and K. Scherer, eds.), Academic Press, New York, 1980, pp. 217-243.

17. H. J. Schmid, On cubature formulae with a minimal number of knots, Numer. Math. 31 (1978), 282-297.

18. _ Interpolatorische Kubaturformeln, Dissertationes Math. (Rozprawy Mat.) 220 (1983), 1-122.

19. __ Minimal cubature formulae and matrix equation, submitted, 1992.

20. I. Sprinkhuizen-Kuyper, Orthogonal polynomials in two variables. A further analysis of the polynomials orthogonal over a region bounded by two lines and a parabola, SIAM J. Math. Anal. 7 (1976), 501-518.

21. Y. Xu, On multivariate orthogonal polynomials, SIAM J. Math. Anal. 24 (1993), 783-794. 
22. __ Multivariate orthogonal polynomials and operator theory, Trans. Amer. Math. Soc. 343 (1994), 193-202.

23. $\ldots$, Gaussian cubature and bivariate polynomial interpolation, Math. Comp. 59 (1992), 547-555.

24. __ Block Jacobi matrices and zeros of multivariate orthogonal polynomials, Trans. Amer. Math. Soc. 342 (1994), 855-866.

25. __ Recurrence formulas for multivariate orthogonal polynomials, Math. Comp. (in print).

26. __ On zeros of multivariate quasi-orthogonal polynomials and Gaussian cubature formulae, SIAM J. Math. Anal. 25 (1994).

Mathematical Institute, University of ERLANGen-Nuremberg, D 91054 ERLangen, GerMANY

E-mail address: schmid@mi. uni-erlangen.de

Department of Mathematics, University of Oregon, Eugene, Oregon 97403

E-mail address: yuan@bright.uoregon.edu 